\title{
The effect of ornithine ingestion on carbohydrate metabolism during rest after acute resistance exercise in healthy young males
}

\author{
Koji Morishita $^{{ }^{*}}$, Shinichi Demura ${ }^{2}$, Takayoshi Yamada ${ }^{3}$, Shunsuke Yamaji ${ }^{4}$, Mami Aoki ${ }^{1}$, \\ Tamotsu Kitabayashi ${ }^{5}$, Masanobu Uchiyama ${ }^{6}$
}

${ }^{1}$ Kyowa Hakko Bio Co., Ltd., Tsukuba, Japan;
${ }^{2}$ Kanazawa University, Kanazawa, Japan;
${ }^{3}$ Fukui National College of Technology, Fukui, Japan;
${ }^{4}$ University of Fukui, Fukui, Japan;
${ }^{5}$ Tokyo University of Science, Tokyo, Japan;
${ }^{6}$ Akita Prefectural University, Akita, Japan.
Email: "koji.morishita@kyowa-kirin.co.jp

Received 6 June 2011; revised 2 July 2011; accepted 11 July 2011.
The effect of oral L-ornithine hydrochloride $(0.1 \mathrm{~g} / \mathrm{kg}$ BW) on energy expenditure during a rest period from 120 to $180 \mathrm{~min}$ after resistance exercise was evaluated by indirect calorimetry. Healthy male subjects who have no habit of resistance training underwent resistance exercise (chest press, lat pulldown, leg press, shoulder press, leg extension, and leg curl), with 3 sets of each exercise and 10 repetitions in each set at 90-s intervals, $30 \mathrm{~min}$ after ingestion of ornithine or placebo. Plasma ornithine levels immediately after, and 120 and $180 \mathrm{~min}$ after, resistance exercise were significantly greater after ingestion of ornithine than of placebo (Treatment: $\mathrm{F}=347.1, P<0.001, \eta_{p}{ }^{2}=0.98$; Time: $\mathrm{F}=175.7, P<0.001, \eta_{p}{ }^{2}=0.95$; Interaction: $F$ $=160.7, P<0.001, \eta_{p}{ }^{2}=0.95$ ), but no significant difference in serum growth hormone levels was observed between the two treatments (Treatment: $F=$ 0.1, $P=0.751, \eta_{p}{ }^{2}=0.01$; Time: $F=1.7, P=0.229, \eta_{p}{ }^{2}$ = 0.16; Interaction: $F=2.4, P=0.155, \eta_{p}{ }^{2}=0.21$ ). Although there was no between-treatment difference in energy expenditure during the rest period 120 to $180 \mathrm{~min}$ after resistance exercise (Treatment: $F=0.1$, $P=0.718, \eta_{p}{ }^{2}=0.02$; Time: $F=0.1, P=0.767, \eta_{p}{ }^{2}=$ 0.01; Interaction: $\mathrm{F}=0.1, P=0.112, \eta_{p}{ }^{2}=0.26$ ), with ornithine ingestion carbohydrate oxidation was significantly greater than with placebo from 170 to 180 min after exercise (Treatment: $\mathrm{F}=0.8, P=0.383, \eta_{p}{ }^{2}$ $=0.09$; Time: $\mathbf{F}=9.7, P=0.013, \eta_{p}{ }^{2}=0.52$; Interaction: $\left.\mathrm{F}=5.8, P=0.039, \eta_{p}{ }^{2}=0.39\right)$. Moreover, 180 min after exercise, serum free fatty acid levels after ornithine ingestion were lower than after placebo (Treatment: $F=0.3, P=0.602, \eta_{p}{ }^{2}=0.03$; Time: $F=$ 34.6, $P<0.001, \eta_{p}{ }^{2}=0.79$; Interaction: $F=5.6, P=$ $0.042, \eta_{p}{ }^{2}=0.38$ ). A similar trend in 3-hydroxybutylate was observed. In conclusion, ornithine ingestion before resistance exercise may enhance post-exercise carbohydrate oxidation without changing total energy expenditure.

Keywords: Resistance Exercise; Ornithine; Carbohydrate Oxidation; Oral; Human

\section{INTRODUCTION}

Many studies have reported that resistance exercise can improve body composition [1,2]. Therefore, resistance exercise is often recommended as a necessary component of exercise programs [3]. Because there is a decrease in resting respiratory exchange ratio after resistance exercise is finished [4-6], lipid oxidation may be increased after this kind of exercise. For $24 \mathrm{~h}$ afterward, resistance exercise has an effect comparable to that of aerobic exercise on total energy expenditure and on the lipid oxidation ratio [7]. Moreover, levels of growth hormone and other endocrine hormones are elevated by resistance exercise [8], and studies of the dynamics of lipid metabolism have evaluated the effects of injected noradrenaline or growth hormone in humans $[9,10]$.

Growth hormone secretion is enhanced not only by resistance exercise or endurance exercise, but also by oral ingestion of amino acids such as glutamine, arginine, or ornithine [11-13]. Recently, we demonstrated that ingestion of ornithine before resistance exercise enhances 
post-exercise growth hormone secretion in healthy male adults [14].

Since it is well known that growth hormone has lipolytic activity, facilitating the release of free fatty acids (FFAs) and glycerol from triglycerides in adipose tissue, we were interested in how orally ingested ornithine affect lipid metabolism during exercise. Since oral ornithine elevated serum growth hormone in the previous study [14], it is expected that ornithine also enhance lipid metabolism. However, there is no definitive evidence to support the hypothesis. Moreover, the availability of practical data on ingestion of ornithine as a sports nutrient is still poor. Here, we aimed to evaluate the effects of ornithine ingestion, especially on energy metabolism, during a period of rest after acute resistance exercise.

\section{MATERIALS \& METHODS}

\subsection{Subjects}

Ten healthy young male students at Kanazawa University who have no habit of resistance training participated in the study (age: $23.2 \pm 1.2$ years; height: $174.3 \pm 5.1$ $\mathrm{cm}$; body-mass: $71.7 \pm 11.9 \mathrm{~kg}$ ). They were habitual participants in sports such as track and field, swimming, soccer, or basketball (3.1 \pm 0.9 times/week, with moderate to high intensity for $2.1 \pm 1.4 \mathrm{~h} /$ time). Written informed consent was obtained from all subjects after a full explanation of the experimental purpose and protocol. The experimental protocol was approved by the Kanazawa University Health and Sports Science Ethics Committee.

\subsection{Experimental Design}

The study used a double-blinded cross-over design. Subjects were given two treatments: L-ornithine hydrochloride (Kyowa Hakko Bio Co., Ltd., Tokyo, Japan) supplementation $(0.1 \mathrm{~g} / \mathrm{kg} \mathrm{BW})$ and placebo (aqueous solution of indigestible dextrin flavored in the same way as the samples containing ornithine), with a washout period of 1 week between treatments. We had the option to use other amino acids as a placebo. However, we were concerned that comparison between ornithine and other amino acids would add a further step to the study. Each subject exercised at the same clock time on the experimental days. Subjects were randomly given ornithine or placebo first to minimize the order effect. In addition, subjects were instructed to refrain from intensive exercise for 2 days before the experiment and fasted from the evening before the experiment. Subjects were also instructed not to consume beverages or food containing caffeine during the experimental period.

\subsection{Exercise Regimen}

The ten-repetition maximum (RM) was measured for chest press, lat pulldown, leg press, shoulder press, leg extension, and leg curl in advance, in accordance with the experimental protocol (Figure 1) to determine the resistance exercise intensity for each subject. Ten repetitions using $50 \%$ to $70 \%$ of the predicted 1RM (onerepetition maximum) were first conducted as a warm-up, and each muscle group was stretched before 10RM measurement. The load was increased until subjects could not complete 10 repetitions, and this was then considered to be the 10RM. Subjects engaged in the experimental protocol (Figure 1) with ornithine or placebo ingestion at least a week after the 10RM measurement. Subjects performed resistance exercise $30 \mathrm{~min}$ after ornithine or placebo ingestion following $60 \mathrm{~min}$ of rest in a semirecumbent position after they had entered the laboratory. Subjects stayed in the semirecumbent rest position for $180 \mathrm{~min}$ after resistance exercise. All experimental protocols started at 8:00 am and finished at 13:00 pm. The resistance exercise was designed in reference to previous studies $[8,15]$. Subjects conducted a chest press, lat pulldown, leg press, shoulder press, leg extension, and leg curl, in that order. Each exercise was composed of three sets of loads. In the first and second sets, the 10RM load determined in advance was performed 10 times. In the third set, subjects repeated each exercise with $85 \%$ of 10RM until exhaustion. There was a 90-s rest after each set. Resistance exercises under both treatment conditions were conducted with the same load and number of repetitions. Hydration (with water) during the experiment was allowed arbitrarily. The laboratory temperature was maintained at $24^{\circ} \mathrm{C}$ to $26^{\circ} \mathrm{C}$.

\subsection{Blood and Expired Gas Analysis}

Initial blood sampling was conducted $60 \mathrm{~min}$ after the start of semirecumbent resting. Other blood samples were taken immediately after resistance exercise and 120 and $180 \mathrm{~min}$ after resistance exercise (Figure 1). Each blood sample $(12 \mathrm{~mL}$ ) was collected from the antecubital vein and used to determine serum levels of growth hormone, FFAs, and 3-hydroxybutylate, and plasma levels of ornithine. Serum samples were placed in chilled containers, and plasma samples were stored frozen at $-80^{\circ} \mathrm{C}$ until analysis. Serum growth hormone was quantified by means of a radioimmunoassay kit (SRL Inc., Tokyo, Japan). The sensitivity and inter-assay and intra-assay coefficients of variation (CVs) in this assay were 0.04

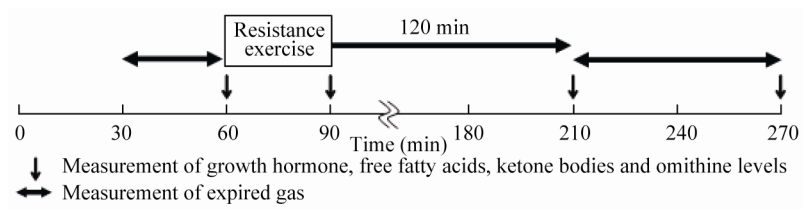

Figure 1. Protocol for exercise and blood sampling. 
$\mathrm{ng} \cdot \mathrm{m} / \mathrm{L}, 4.0 \%$, and $3.4 \%$, respectively. Serum FFAs and 3-hydroxybutylate concentrations were quantified enzymatically; the interassay and intraassay CVs were $0.2 \%$ and $0.9 \%$ for FFAs and $0.6 \%$ and $0.7 \%$ for 3-hydroxybutylate. Plasma levels of ornithine were determined by HPLC (Shimadzu Co., Tokyo, Japan). The sensitivity and inter-assay and intra-assay CVs in this assay were $5.92 \mathrm{nmol} / \mathrm{L}, 4.94 \%$, and $0.00 \%$, respectively. Expired gases were continuously sampled in a breath-by-breath method during the last half of the first 60-min semirecumbent rest period after entry to the laboratory and during the 60-min period from 120 to $180 \mathrm{~m}$ after resistance exercise. Oxygen consumption $\left(\mathrm{VO}_{2}\right)$ and carbon dioxide production $\left(\mathrm{VCO}_{2}\right)$ were measured with an automatic expired gas analysis system (AE-280S; Minato Medical Science Co. Ltd., Osaka, Japan). Subjects remained in the semirecumbent rest position during expired gas sampling. The respiratory exchange ratio (RER) was used to estimate the relative contributions of fat and carbohydrate oxidation to total energy production [16]. RER and percentage fat oxidation were determined without urinary nitrogen analysis because of the negligible contribution of protein to substrate oxidation during rest [17].

\subsection{Statistical Analysis}

Two-way repeated-measures analysis of variance (treatment $\times$ measurement time) was used to examine the effect of ornithine ingestion. When there was a significant main or interaction effect, Tukey's honestly significant difference was used in a post-hoc analysis to examine specific mean differences. An alpha concentration of 0.05 was used for all tests.

\section{RESULTS}

Levels of plasma ornithine with ornithine ingestion were significantly elevated at all times after exercise compared with before and were significantly higher than with placebo ingestion immediately after exercise and 120 and $180 \mathrm{~min}$ after exercise (Treatment: $\mathrm{F}=347.1, P$ $<0.001, \eta_{p}{ }^{2}=0.98$; Time: $\mathrm{F}=175.7, P<0.001, \eta_{p}{ }^{2}=$ 0.95; Interaction: $\left.\mathrm{F}=160.7, P<0.001, \eta_{p}{ }^{2}=0.95\right)$ (Figure 2). However, there was no significant change in growth hormone concentration after resistance exercise with either treatment, and there was no significant difference between the two treatments (Treatment: $\mathrm{F}=0.1$, $P=0.751, \eta_{p}{ }^{2}=0.01$; Time: $\mathrm{F}=1.7, P=0.229, \eta_{p}{ }^{2}=$ 0.16; Interaction: $\mathrm{F}=2.4, P=0.155, \eta_{p}{ }^{2}=0.21$ ) (Figure 3). With placebo treatment, the serum FFA concentration was significantly greater $180 \mathrm{~min}$ after exercise than before exercise or immediately after exercise; at $180 \mathrm{~min}$ after exercise it was also significantly greater than after ornithine treatment (Treatment: $\mathrm{F}=1.7, P=0.219, \eta_{p}{ }^{2}=$

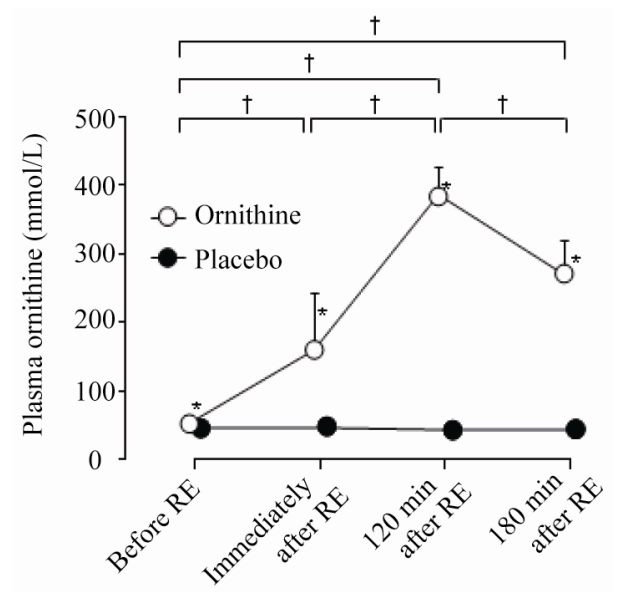

Figure 2. Plasma ornithine levels under the two treatments. Values are means $\pm \mathrm{SD}$. RE, resistance exercise; *significant difference between treatments; †significant difference between measurements under ornithine conditions.

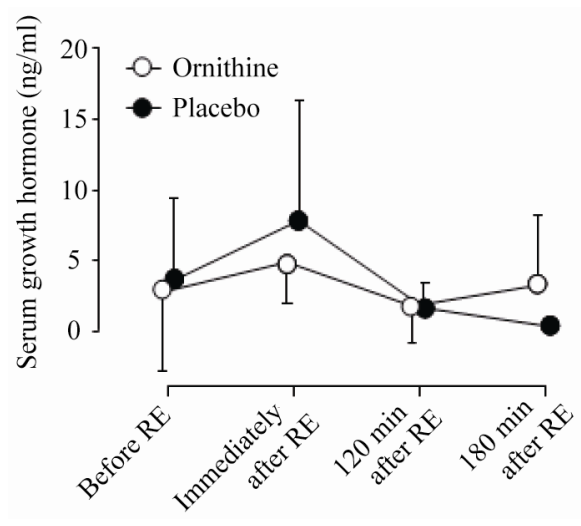

Figure 3. Serum growth hormone levels under the two treatments. Values are means \pm SD. RE, resistance exercise.

0.16; Time: $\mathrm{F}=25.0, P=0.001, \eta_{p}{ }^{2}=0.74$; Interaction: $\left.\mathrm{F}=6.6, P=0.003, \eta_{p}{ }^{2}=0.65\right)$. Similar results were found for serum ketone body, 3-hydroxybutyrate (Treatment: $\mathrm{F}=2.0, P=0.187, \eta_{p}^{2}=0.18$; Time: $\mathrm{F}=12.0, P=$ $0.007, \eta_{p}{ }^{2}=0.57$; Interaction: $\mathrm{F}=8.5, P=0.019, \eta_{p}{ }^{2}=$ 0.49) (Figure 4). Fat oxidation as a percentage of total energy expenditure with ornithine treatment decreased after the start of post-exercise respiratory gas analysis (120 min after exercise) and was significantly lower 170 to $180 \mathrm{~min}$ after exercise than $120 \mathrm{~min}$ after (Treatment: $\mathrm{F}=0.2, P=0.707, \eta_{p}{ }^{2}=0.02$; Time: $\mathrm{F}=15.1, P=0.004$, $\eta_{p}{ }^{2}=0.63$; Interaction: $\mathrm{F}=3.1, P=0.112, \eta_{p}{ }^{2}=0.26$ ). From 170 to $180 \mathrm{~min}$ after resistance exercise, the percentage fat oxidation with ornithine treatment was significantly lower than with placebo treatment (Figure 5 and 6) and the percentage carbohydrate oxidation with or- 


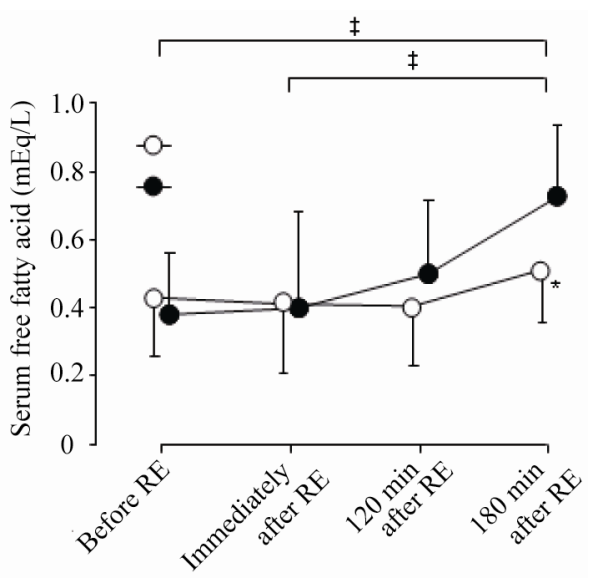

Figure 4. Serum free fatty acid levels under the two treatments. Values are means $\pm \mathrm{SD}$. $\mathrm{RE}$, resistance exercise; ${ }^{\text {significant difference }}$ between the two treatments; fsignificant difference between measurements under placebo conditions.

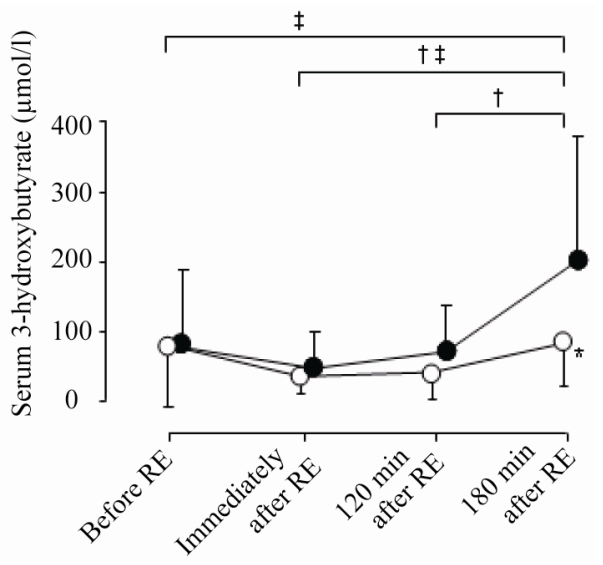

Figure 5. Serum 3-hydroxybutyrate levels under the two treatments. Values are means \pm SD. $\mathrm{RE}$, resistance exercise; *significant difference between the two treatments; †significant difference between measurements under ornithine conditions; \$significant difference between measurements under placebo conditions.

nithine treatment was significantly higher than with placebo treatment (Treatment: $\mathrm{F}=0.8, P=0.383, \eta_{p}{ }^{2}=0.09$; Time: $\mathrm{F}=9.7, P=0.013, \eta_{p}{ }^{2}=0.52$; Interaction: $\mathrm{F}=5.8$, $P=0.039, \eta_{p}{ }^{2}=0.39$ ) (Figure 7), However, total energy expenditure at all the times did not differ between the two treatments (Treatment: $\mathrm{F}=0.1, P=0.718, \eta_{p}{ }^{2}=0.02$; Time: $\mathrm{F}=0.1, P=0.767, \eta_{p}{ }^{2}=0.01$; Interaction: $\mathrm{F}=0.1$, $\left.P=0.112, \eta_{p}{ }^{2}=0.26\right)$ (Figure 8).

\section{DISCUSSION}

We evaluated the effect of oral ornithine on energy metabolism during rest after resistance exercise by means

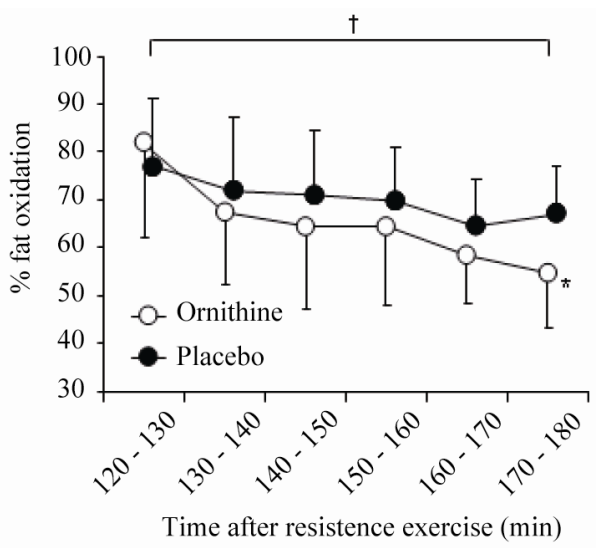

Figure 6. Relative contributions of fat oxidation (\%) to total energy expenditure 120 to 180 min after resistance exercise under the two treatments. Values are means $\pm \mathrm{SD} .{ }^{*}$ significant difference between the two treatments; †significant difference between measurements under ornithine conditions.

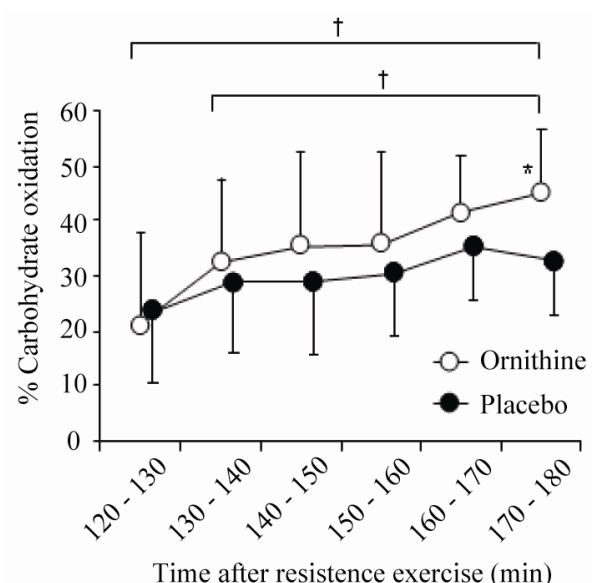

Figure 7. Relative contributions of carbohydrate oxidation (\%) to total energy expenditure 120 to $180 \mathrm{~min}$ after resistance exercise under the two treatments. Values are means \pm SD. *significant difference between the two treatments; †significant difference between measurements under ornithine conditions.

of indirect calorimetry. Although the involvement of growth hormone in the lipolysis that follows resistance exercise is still unclear, the occurrence of lipolysis after resistance exercise has been confirmed in some studies [4-7]. Meanwhile, injected growth hormone shows clear lipolytic activity in humans $[9,18]$, and orally ingested ornithine can elevate serum levels of growth hormone [11]. Moreover, we recently reported that oral L-ornithine hydrochloride $(100 \mathrm{mg} / \mathrm{kg} \mathrm{BW})$ can elevate serum growth hormone levels after resistance exercise [14]. It can therefore be hypothesized that oral ornithine en- 
hances lipid oxidation and increases the levels of metabolites of fatty acid oxidation through the activity of growth hormone, which is a lipolytic hormone. However, here we obtained the opposite result, i.e. a reduction in percentage fat oxidation and in the serum levels of FFAs and their metabolites. Therefore, it is possible that oral ornithine enhances carbohydrate oxidation after resistance exercise.

Indirect calorimetry is a non-invasive technique used to evaluate substrate contribution to total energy expenditure; therefore, it is used widely in studies of sports nutrition [19], although this method has a limitation in that physiological or biochemical conditions such as hyperventilation or metabolic acidosis can shift RER values to higher levels [20]. However we have no reason that there were any factors to induce hyperventilation or metabolic acidosis. It is therefore reasonable to suggest that carbohydrate metabolism was enhanced by ornithine treatment.

With ornithine ingestion before resistance exercise, the plasma ornithine concentration increased significantly after exercise (Figure 2). However, with ornithine treatment the serum growth hormone levels were not higher than with placebo (Figure 3). The elevations in growth hormone that we observed recently in another study occurred $30 \mathrm{~min}$ after resistance exercise following ornithine ingestion [14]. It has previously been reported that growth hormone levels peak 15 min after resistance exercise [8]. Moreover, significant increases in serum growth hormone concentration have been observed $90 \mathrm{~min}$ after exercise in bodybuilders [11]. As indicated above, the amount of time between ornithine ingestion and the rise in growth hormone level varies among studies; in the previous studies growth hormone was quantified earlier than $120 \mathrm{~min}$ after exercise, whereas we did not begin our post-exercise respiratory gas analysis until $120 \mathrm{~min}$. Therefore, the lack of increase in growth hormone level in our study does not mean that growth hormone levels did not increase after resistance exercise with oral ornithine.

It is well recognized that growth hormone can shift the energy balance to energy supply, i.e. catabolism, not only with lipid oxidation but also with carbohydrate oxidation via glycogenolysis. However, injected growth hormone induces a lipolytic response, the onset of which is delayed by approximately $2 \mathrm{~h}$ after the injection [18]. It is therefore reasonable that we focused on lipid oxidation by oral ornithine via growth hormone and that the timing chosen for our respiratory gas analysis was 120 min after exercise. In fact, elevated serum metabolites of fatty acid oxidation were observed with placebo treatment at $120 \mathrm{~min}$, and a significant increase occurred 180 min after exercise compared with before exercise (Fig-

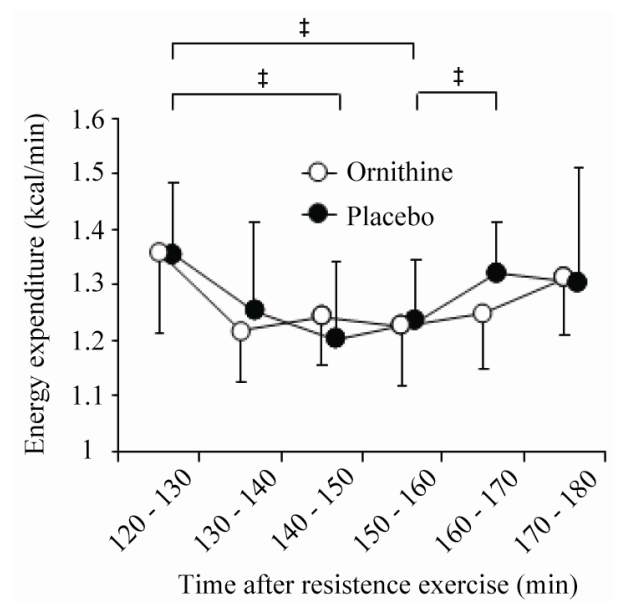

Figure 8. Energy expenditure 120 to $180 \mathrm{~min}$ after resistance exercise under the two treatments. Values are means $\pm \mathrm{SD}$. $\$$ significant difference between measurements under placebo conditions.

ure 8).

Unfortunately these findings in the present study can no practical application directly. It is expected that more carefully designed studies can unveil benefits of oral ornithine via growth hormone for sports nutrition.

\section{CONCLUSIONS}

Ingestion of ornithine by healthy males before resistance exercise may enhance carbohydrate oxidation as a percentage of total energy expenditure under post-exercise resting conditions.

\section{REFERENCES}

[1] Chatzinikolaou, A., Fatouros, I., Petridou, A., et al. (2008) Adipose tissue lipolysis is upregulated in lean and obese men during acute resistance exercise. Diabetes Care, 31, 1397-1399. doi:10.2337/dc08-0072

[2] Yarasheski, K.E., (2008) Review article: Exercise, aging, and muscle protein metabolism. The Journals of Gerontology, Series A: Biological Sciences, 58, M918-M922.

[3] Pollock, M., Gaesser, G., Butcher, J., et al. (1998) American College of Sports Medicine Position Stand. The recommended quantity and quality of exercise for developing and maintaining cardiorespiratory and muscular fitness, and flexibility in healthy adults. Medicine \& Science in Sports \& Exercise, 30, 975-991. doi:10.1097/00005768-199806000-00032

[4] Binzen, C., Swan, P. and Manore, M. (2001) Postexercise oxygen consumption and substrate use after resistance exercise in women. Medicine \& Science in Sports \& Exercise, 33, 932-938. doi:10.1097/00005768-200106000-00012

[5] Melby, C., Scholl, C., Edwards, G., et al. (1993) Effect of acute resistance exercise on postexercise energy expenditure and resting metabolic rate. Journal of Applied 
Physiology, 75, 1847-1853.

[6] Ormsbee, M., Thyfault, J., Johnson, E., et al. (2007) Fat metabolism and acute resistance exercise in trained men. Journal of Applied Physiology, 102, 1767-1772. doi:10.1152/japplphysiol.00704.2006

[7] Melanson, E., Sharp, T., Seagle, H., et al. (2002) Resistance and aerobic exercise have similar effects on 24-h nutrient oxidation. Medicine \& Science in Sports \& Exercise, 34, 1793-1800. doi:10.1097/00005768-200211000-00016

[8] Kraemer, W.J., Marchitelli, L., Gordon, S.E., et al. (1990) Hormonal and growth factor responses to heavy resistance exercise protocols. Journal of Applied Physiology, 69, 1442-1450.

[9] Møller, N., Jørgensen, J.O., Alberti, K.G., et al. (1990) Short-term effects of growth hormone on fuel oxidation and regional substrate metabolism in normal man. The Journal of Clinical Endocrinology \& Metabolism, 70, 1179-1186.

[10] Quisth, V., Enoksson, S., Blaak, E., et al. (2005) Major differences in noradrenaline action on lipolysis and blood flow rates in skeletal muscle and adipose tissue in vivo. Diabetologia, 48, 946-953. doi:10.1007/s00125-005-1708-4

[11] Bucci, L., Hickson, J.F., Pivarnik, J.M., et al. (1990) Ornithine ingestion and growth hormone release in bodybuilders. Nutrition Research, 10, 239-245. doi:10.1016/S0271-5317(05)80265-9

[12] Welbourne, T.C. (1995) Increased plasma bicarbonate and growth hormone after an oral glutamine load. American Journal of Clinical Nutrition, 61, 1058-1061

[13] Besset, A., Bonardet, A., Rondouin, G., et al. (1982) Increase in sleep related GH and Prl secretion after chronic arginine aspartate administration in man. Acta Endocrinologica, 99, 18-23.

[14] Demura, S., Yamada, T., Yamaji, S., et al. (2010) The effect of L-ornithine hydrochloride ingestion on human growth hormone secretion after strength training. $A d$ vances in Bioscience and Biotechnology, 1, 7-11. doi:10.4236/abb.2010.11002

[15] Thyfault, J.P., Carper, M.J., Richmond, S.R., et al. (2004) Effects of liquid carbohydrate ingestion on markers of anabolism following high-intensity resistance exercise. The Journal of Strength \& Conditioning Research, 18, 174-179.

[16] Manetta, J., Brun, J.F., Perez-Martin, A., et al. (2002) Fuel oxidation during exercise in middle-aged men: Role of training and glucose disposal. Medicine \& Science in Sports \& Exercise, 34, 423-429. doi:10.1097/00005768-200203000-00007

[17] Beidleman, B.A., Rock, P.B., Muza, S.R., et al. (2002) Substrate oxidation is altered in women during exercise upon acute altitude exposure. Medicine \& Science in Sports \& Exercise, 34, 430-437. doi:10.1097/00005768-200203000-00008

[18] Gravhølt, C.H., Schmitz, O., Simonsen, L., et al. (1999) Effects of a physiological GH pulse on interstitial glycerol in abdominal and femoral adipose tissue. American Journal of Physiology, 277, E848-E854.

[19] Ferrannini, E. (1899) The theoretical bases of indirect calorimetry: A review. Metabolism, 37, 287-301. doi:10.1016/0026-0495(88)90110-2

[20] Jéquier, E. and Felber, J. (1987) 7 Indirect calorimetry. Baillière's Clinical Endocrinology and Metabolism, 1, 911-935. 